Meta

Journal des traducteurs

Translators' Journal

\title{
Réflexions sur le discours juridique
}

\section{Jean Darbelnet}

Volume 24, numéro 1, mars 1979

La traduction juridique

URI : https://id.erudit.org/iderudit/002480ar

DOI : https://doi.org/10.7202/002480ar

Aller au sommaire du numéro

Éditeur(s)

Les Presses de l'Université de Montréal

ISSN

0026-0452 (imprimé)

1492-1421 (numérique)

Découvrir la revue

Citer cet article

Darbelnet, J. (1979). Réflexions sur le discours juridique. Meta, 24(1), 26-34.

https://doi.org/10.7202/002480ar d'utilisation que vous pouvez consulter en ligne.

https://apropos.erudit.org/fr/usagers/politique-dutilisation/ 


\section{Réflexions sur le discours juridique}

Tout texte, tout message, oral ou parlé, est fait de mots agencés selon certains schèmes que la langue fournit à l'usager. Il faut comprendre que la signification du message dépend nécessairement et du choix des mots et de leur agencement, et il serait vain de chercher à établir un ordre d'importance entre ces deux composantes du texte ainsi réalisé. En d'autres termes, le sens que véhiculent les mots du texte, si bien choisis qu'ils soient, ne passera pas si ces mots ne sont pas agencés et articulés d'une certaine façon. Cela est vrai même du style télégraphique dont l'abrègement est toujours calculé pour laisser passer le sens. Tout cela est évident, mais il importait de le rappeler pour dégager l'interaction de ces deux éléments linguistiques que sont d'une part la nomenclature du sujet traité et d'autre part le vocabulaire qui encadre les termes et assure leur enchaînement.

Nomenclature est pris ici dans son acception reconnue de liste de termes particuliers à un sujet. Par vocabulaire de soutien, qui sert à encadrer les termes techniques, nous entendons les mots qui, étant d'une technicité moindre ou nulle, servent à actualiser les mots spécialisés et à donner ainsi au texte son organicité. Dans la perspective où nous nous plaçons, le discours comprend essentiellement le vocabulaire de soutien, cette notion impliquant la liaison aussi bien que le soutien. Les éléments stylistiques tels que le choix des mots de soutien et de liaison (les termes techniques étant, eux, imposés par le sujet) et leur mise en valeur, par exemple à des fins dialectiques, relèvent aussi du discours.

La notion de terme technique est généralement appliquée à des mots qui, familiers au spécialiste, présentent pour le profane un aspect rébarbatif et hermétique. Il convient cependant d'inclure dans cette notion des mots usuels mais ayant un sens technique. Il y a là, pour la terminologie, une distinction importante entre la forme, qui ne tranche pas sur le vocabulaire usuel, et le sens, qui est technique. Ainsi long est un adjectif banal dans une «longue journée », il est technique dans le terme de navigation au long cours. Quand le général de Gaulle, dans la $2^{\circ}$ édition de Vers l'armée de métier, mentionne les vertus de rupture et d'exploitation des grandes unités mécaniques, il emploie trois termes (ici soulignés) du vocabulaire courant que tout le monde connaît - ou croit connaître, car en fait la langue militaire leur donne un sens très précis qui n'est pas le sens usuel. Cet exemple a été pris à dessein en dehors du domaine juri- 
dique pour rappeler que chaque spécialité a son discours, ne peut être appréhendée que par son discours.

Nous verrons que certains éléments du vocabulaire de soutien empruntent à leurs contextes une valeur technique de ce genre. La distinction n'est donc pas toujours tranchée entre mots techniques et mots de soutien. La cohésion du discours est à ce prix.

S'il convient d'insister sur l'existence du vocabulaire de soutien, c'est parce que son importance est éclipsée, dans les répertoires spécialisés, par les termes techniques. On constate, en feuilletant des répertoires de la langue juridique, par exemple le Lexique de termes juridiques, qui pourtant s'adresse à des étudiants, que nombre de termes utilisés dans la rédaction de textes juridiques et administratifs (ces deux domaines communiquent largement) n'ont pas été retenus. Il est vrai qu'en général les répertoires visent plutôt à la compréhension qu'à la rédaction. C'est pourquoi on y trouve des mots comme syndic, réclusion, parafiscalité, ou encore défendeur, inculpé, prévenu et accusé, mais sans que, soit dit en passant, le LTJ montre le lien entre ces quatre derniers termes. Le sens de tous ces mots est facile à cerner et le relief que cela leur donne explique sans doute leur inclusion. En revanche on risque de ne pas rencontrer dans les mêmes répertoires des mots de soutien, tels que intenter, entendre (un témoin, le Conseil d'État entendu) exorbiter, dépérir, supporter (au sens fiscal). La notion de discours, telle que nous venons de la définir, permet de faire ressortir l'importance de ce vocabulaire paratechnique dans la terminologie du droit et l'utilité que présente son étude. Il apparât comme marginal, il n'en est pas moins indispensable, si l'on veut avoir une vue suffisamment détaillée des ressources lexicales et syntaxiques qu'offre la langue pour traiter un sujet technique. Mais on comprend que cet aspect ait été négligé par les lexicographes terminologues au profit des termes techniques proprement dits, c'est-à-dire ceux qui par leur forme et leur contenu rationnel frappent comme étant à la fois essentiel et évident. Par rapport à ces éléments d'une nomenclature technique, le vocabulaire de soutien tel que nous l'avons défini, semble n'avoir qu'un rôle effacé. Cela tient en partie à sa relative compréhensibilité par rapport aux termes qu'il encadre. On peut dire que le terme technique, opaque pour le profane, ne devient transparent que si on possède une connaissance exacte de la réalité qu'il recouvre. Ce résultat atteint, il est d'un maniement facile. Antithétiquement, les mots de soutien sont, ou paraissent transparents, mais ils sont d'un maniement plus délicat, comme le montre le cas d'ingénieurs dont les rapports manquent parfois de clarté, tout au moins pour les destinataires, alors que de toute évidence ces ingénieurs connaissent parfaitement leur spécialité et sa terminologie.

Certains chercheurs ont bien vu cette dichotomie de la nomenclature et de son entourage, que l'on peut appeler rédactionnel, car il est fait des mots et des tours dont on a besoin pour passer d'une simple liste à un texte explicatif et par conséquent discursif. C'est au moment où il faut rédiger que le vocabulaire de soutien prend toute sa valeur. 
À l'École normale supérieure de Saint-Cloud a été étudié dans cette optique, il y a quelques années, le vocabulaire des manuels scolaires du niveau secondaire dans les différentes disciplines. L'un des objectifs de cette recherche était de mieux préparer les étudiants étrangers à l'acquisition du français scientifique. (En fait ce problème se pose aussi, mais avec une acuité moindre, pour l'étudiant qui utilise sa langue maternelle.) Une première étape avait permis de constater que, contrairement à ce que l'on attendait, ce n'était pas l'étude de telle ou telle terminologie qui constituait le problème le plus important. Dès l'instant que l'étudiant en question doit s'exprimer oralement ou par écrit sur un sujet scientifique, on peut dire que des deux besoins cités plus haut, le plus pressant et le moins facile à satisfaire est celui de l'élocution. Il importe donc qu'il développe sa faculté d'élocution, qui justement fait appel au vocabulaire d'encadrement que les chercheurs de Saint-Cloud ont appelé le vocabulaire général d'orientation scientifique (VGOS), expression qui dit bien ce qu'elle veut dire puisqu'il s'agit de manuels d'initiation à des disciplines d'ordre scientifique et qui, de par leur diversité, présentent globalement un caractère de généralité. Le terme «vocabulaire de soutien» peut être considéré comme équivalent, et convient mieux à la perspective dans laquelle nous nous plaçons ici. Voici quelques exemples de VGOS donnés par les chercheurs de Saint-Cloud aux fins de démonstration. Ce sont des cas d'équivalence entre la langue usuelle et la langue scientifique. Certains sont des plus élémentaires : bout et extrémité, se faire et s'effectuer. D'autres sont moins évidents. Le profane dira que l'acide ronge le métal, tandis que, pour le spécialiste, il l'attaque. Le médecin voit son malade à intervalles plus ou moins rapprochés pour s'assurer de l'efficacité du traitement prescrit. Cette idée du contact que le médecin garde avec son malade et que nous venons de définir en une quinzaine de mots, peut se rendre par un seul mot très simple de la langue la plus usuelle : le médecin suit son malade. Soit dit en passant, c'est ce sens de suivre, étendu à d'autres domaines, que nous trouvons dans le néologisme suivi (le suivi d'une affaire). Nous pouvons dire en guise de conclusion et pour revenir à notre schème, qu'à SaintCloud, on a jugé le discours plus important que la nomenclature. $\AA$ la réflexion on se dit que la réalité confirme ce jugement.

La notion de discours dont nous avons entrepris de montrer comment elle s'applique à la langue juridique est donc, comme nous venons de le voir, mais sous une autre appellation, valable pour d'autres domaines techniques. Le terme discours paraît préférable parce qu'il est encore plus général que vocabulaire général d'orientation scientifique, même si l'on tient compte du fait que la littérature et l'histoire peuvent être étudiées dans un esprit scientifique. En outre, VGOS ne renvoie qu'au vocabulaire, alors que le discours, tel que nous le concevons, comporte d'autres éléments que les mots de soutien, la syntaxe et même la disposition matérielle d'un énoncé étant aussi des faits de discours.

Ainsi, dans une étude grammaticale des temps narratifs du passé en français, on peut fort bien présenter le passé simple ou prétérit comme le temps passé de deux discours, à savoir le discours romanesque et le discours historique. $\mathrm{Du}$ moins on le peut encore aujourd'hui. C'est encore vrai dans l'ensemble, 
malgré l'emploi du passé composé dans ce récit qu'est l'Étranger de Camus et dans des ouvrages de vulgarisation consacrée à l'histoire moderne.

On peut aussi dans le domaine des modes et temps de verbe considérer l'écart stylistique que produit l'alternance entre l'infinitif et l'impératif dans le discours qu'adresse au candidat le questionnaire d'un examen. Le français a en effet le choix - refusé à l'anglais - entre la directive à l'infinitif et celle qui utilise l'impératif : Répondre ou Répondez aux questions suivantes. La tendance actuelle qui incite l'Administration à établir un contact personnel avec le public, semble préférer l'impératif. Sur ce point, le changement est encore plus frappant dans le cas des feuilles d'impôts. D'une façon générale, on peut dire que nous sortons de l'ère de l'imprimé à l'infinitif pour entrer dans celle de l'imprimé à l'impératif. L'impératif est, évidemment, à la deuxième personne du pluriel, mais cela peut changer. Déjà dans certaines banques du Québec un avis concis et percutant rappelle au client, en le tutoyant, qu'il a intérêt à faire telle démarche. C'est dans la ligne du \& As-tu donné ton deux ? \&ue beaucoup de Montréalais se rappellent encore.

Et puisque nous parlons grammaire, n'oublions pas que la grammaire, matière d'enseignement, donne lieu à un discours grammatical, même si la division des pages en paragraphes avec sous-titres marginaux produit un aspect de fragmentation que l'on n'associe pas d'ordinaire avec l'idée de discours. Mais le dictionnaire lui-même, dont les définitions n'ont parfois que quelques lignes, constitue un discours sur les mots. Et d'ailleurs ce que la linguistique moderne appelle « métalangage » n'est pas autre chose qu'un discours sur la langue. Certains ouvrages se rapprochent plus que d'autres du discours sous la forme que ce mot évoque. Ainsi le ton « discours » est plus apparent chez Fischer et Hacquard ( $A$ la découverte de la grammaire française) que chez Grevisse, mais c'est surtout le Comment on parle en français, de $\mathrm{Ph}$. Martinon, qui se présente comme un discours aussi bien par le ton que par l'absence de paragraphes titrés. Nous avons déjà fait observer que la typographie relève du discours, et c'est une raison de plus de distinguer entre style et discours, si rapprochés par le sens que soient ces deux termes.

L'occasion a été donnée récemment à l'auteur de ces lignes de prendre connaissance d'un projet de manuel élémentaire pour l'enseignement de la géographie. Il a pu se rendre compte que le discours géographique diffère notablement de celui qu'il a connu comme élève. Et d'abord par le ton, indépendamment du choix de mots. Il s'agit en effet d'un discours très personnalisé qui s'adresse à l'élève, et non aux élèves, et qui tutoie cet élève. La question du tutoiement est un phénomène infiniment complexe, en français tout au moins, dès qu'on passe aux cas particuliers. Ce que nous relevons ici c'est la forme imprimée du tutoiement, car il est probable que même jadis, dans les écoles de campagne, beaucoup d'instituteurs tutoyaient leurs élèves. Pourtant il convient de noter en passant que dans un livre lu par plusieurs générations d'élèves de l'école primaire entre 1877 et 1914, le Tour de la France par deux enfants, une vieille dame qui héberge ces deux enfants, dont le plus jeune a sept ans, les vouvoie tous les deux. 
Conformément à l'esprit de la pédagogie moderne qui cherche à diminuer ou même à supprimer les distances entre enseignants et enseignés, l'illustration du manuel projeté doit comporter des éléments de bandes dessinées dont l'effet s'ajoute à celui du tutoiement et renforce le ton de camaraderie sur lequel s'établit le contact entre le maître et l'élève. Ce détail de présentation est significatif et fait partie, lui aussi, du discours.

Les discours varient non seulement en fonction des domaines dont ils traitent, c'est-à-dire de ce qu'on pourrait appeler leur contenu technique, mais aussi par leurs choix syntaxiques et comme on vient d'en avoir un exemple, par des détails de présentation. C'est ainsi que le discours didactique peut refléter la pédagogie de son temps.

On peut s'attendre à plus de réserve vis-à-vis de l'usager quand on a affaire à l'Administration ou à la justice, dont les domaines et, partant, les discours sont très proches, au point parfois de se confondre. Ces deux institutions réglementent en effet la vie du citoyen et lui parlent à peu près sur le même ton. L'autorité que cette vocation leur confère se traduit dans leurs discours respectifs par une dignité de langage qui atteint par moments à la solennité. C'est pourquoi on ne peut s'attendre, malgré de récentes initiatives auxquelles il sera fait allusion plus loin, à des changements importants dans le discours que l'État adresse à ses administrés par la voix de ces deux institutions.

Nous possédons, sur le côté administratif de ce discours, un petit livre qui fait autorité dans ce domaine parce qu'il combine heureusement la théorie et la pratique grâce à la richesse des exemples qui viennent se greffer sur une solide base théorique. C'est le Style administratif, de Robert Catherine, dont le titre fournit un autre exemple de la synonymie style/discours déjà signalée.

Mutatis mutandis, on retrouve dans ce discours les catégories d'éléments déjà notées dans les discours didactiques, à savoir les termes particuliers aux sujets traités, un vocabulaire de soutien, dont Catherine donne un bon répertoire, et, sur certains points, des faits de syntaxe.

Comme exemple de vocabulaire de soutien, on peut considérer les trois tournures dont l'Administration use quand elle a quelque chose à communiquer. Son formalisme bien connu apparaît dans son souci de distinguer entre les trois niveaux de destinataires possibles : le supérieur, l'égal et l'inférieur. Cela donne, dans cet ordre, les formes rendre compte, informer, et faire connaître. Cette dernière expression a une variante, faire savoir, qui accentue la distance entre le supérieur et l'inférieur. De la même façon, on distingue entre «bien vouloir 》 (je vous prie de bien vouloir) qui fait appel au bon vouloir, et «vouloir bien » qui semble impliquer la soumission. On s'est moqué de ces distinctions subtiles, mais il n'est pas sûr que si on réussissait à les supprimer on ne serait pas amené à les rétablir plus tard sous une autre forme. Leur justification repose sur la sensibilité de ceux par qui et avec qui elles sont employées, or c'est un fait que la sensibilité langagière évolue tout comme la sensibilité littéraire, mais avec plus de lenteur, les formes dụ langage étant plus contraignantes que les modes littéraires. Ces distinctions entrent dans la catégorie des faits de culture, dont on 
sait qu'ils sont essentiellement relatifs. D'autres époques, d'autres cultures pourraient ne pas les connaître. En particulier, le traducteur vers l'anglais doit se demander si la langue d'arrivée les pratique, avant de se préoccuper de les reproduire littéralement.

Dans le domaine de la syntaxe, on note l'emploi fréquent du passif, parfois étendu au mode impersonnel ( il est tenu procès-verbal des séances ») et l'absence du pronom impersonnel on que remplace justement le passif impersonnel dans certains cas. La tournure « on tiendra procès-verbal des séances » peut être grammaticale, elle n'est pas idiomatique dans ce genre de discours. Les universitaires savent que l'annuaire de leur faculté n'emploierait pas on de cette façon. Autre particularité du discours administratif ou juridique, l'absence du point d'interrogation. L'Administration ne condescend pas à poser de questions et la Justice n'en a pas l'occasion. On n'écrira pas «Y a-t-il lieu de... » mais \& La question se pose de savoir si..." ou encore «Je vous serais obligé (et non « reconnaissant $》)$ de bien vouloir m'indiquer si... »C'est donc à l'interrogation indirecte qu'on a recours pour obtenir une précision.

On notera que ces caractéristiques de syntaxe passent très bien en anglais, cette langue faisant un large usage du passif, qui limite, sur ce point, l'emploi $\mathrm{du}$ pronom one.

Le discours juridique dispose naturellement d'un très grand nombre de termes techniques que seul le juriste peut manier avec sûreté, puisque c'est une caractéristique de tout terme technique de n'être compris que si on connaît de première main la réalité qu'il recouvre, ce qui exige naturellement une formation spécialisée.

Dans le vocabulaire de soutien, on retrouve la catégorie des mots appartenant à la langue usuelle, mais qui ont pris un sens technique. Ils peuvent gêner ou égarer la compréhension tout comme un terme technique. Lorsqu'un ministre français fut accusé, il y a quelques années, de ne pas avoir payé tous ses impôts, un communiqué du ministère des Finances donna le montant des impôts qu'avait «supportés » le ministre au cours de trois années précédentes. Peu de gens, même dans les milieux bien informés, comprirent que ces impôts «supportés» avaient en fait été prélevés à la source.

Une autre catégorie est celle des termes qui découpent la réalité différemment dans deux langues données, et il arrive parfois qu'il y ait en fait deux réalités au lieu d'une. Il existe des mots de ce genre dans le vocabulaire le plus courant; c'est-à-dire que tout le monde emploie. Libel permet de faire en anglais une distinction que le français ne connait pas. Par contre nous distinguons sans équivoque possible le droit et la loi. Il est vrai que dans une certaine mesure l'anglais fait de même en actualisant law différemment : He studied law/the law. Plus intéressante et plus souvent négligée par les anglophones est la différence que tout Français fait entre l'État et le gouvernement. Sans doute on peut dire the State, state-owned, state-controlled en anglais, mais ce sera surtout en anglais britannique, l'anglais américain appliquant généralement state à l'un des 50 États dë l'Union. Il en résulte que l'Américain moyen emploie couram- 
ment the Government dans des cas où le Français dirait l'Etat, car pour lui le gouvernement c'est seulement l'instrument du pouvoir exécutif, l'ensemble des ministres auxquels le parlement pourrait retirer sa confiance. C'est l'État, et non le gouvernement, pour ne citer qu'un exemple, qui émet les billets de banque. Ce sont là des distinctions auxquelles tout traducteur doit prendre garde. Il doit aussi savoir qu'en France, cour se dit des juridictions supérieures à celles des tribunaux et que, pour un anglophone, le juge et le magistrat sont à des niveaux différents de l'appareil judiciaire.

On sait l'importance de la ponctuation dans un texe juridique. Cete remarque vaut également pour les mots de liaison (par exemple, la différence entre aussi indiquant la conséquence et aussi indiquant l'addition) et même pour de simples adverbes qui peuvent, s'ils sont mal employés, altérer la valeur du segment d'énoncé qu'ils modifient. Cela a été le cas de notamment, qu'à une séance de la Commission de l'énergie atomique de l'ONU l'interprète avait traduit par mainly. Le représentant du Royaume-Uni protesta et obtint que la traduction fût corrigée en remplaçant mainly par in particular.

Si maintenant on considère l'anglais, on remarque que bien souvent son emploi fréquent de all, any, such dans les textes juridiques ne se retrouve pas dans une traduction idiomatique. Les phrases suivantes sont traduites de l'anglais. La traduction littérale du mot donné chaque fois entre parenthèses a été omise sans que le sens en souffre.

- Prière de signaler les conflits d'horaire (all).

- Lorsqu'un membre du corps enseignant... (any).

- Le Comité prend les mesures que... (such... as).

Dans l'ensemble, le discours juridique est plus dépouillé en anglais qu'en français ${ }^{1}$.

En 1977, le ministre français de la Justice fit état dans une circulaire des recommandations de la Commission de modernisation du langage judiciaire. Il n'y est pas touché a-t-il dit, aux termes techniques. Les changements proposés concernent à peu près uniquement ce que nous appelons ici des faits de discours : les locutions latines, les expressions étrangères, archaïques, surannées, discourtoises, inutiles ou creuses, inintelligibles ou ambiguës.

On voit bien que dans le contexte actuel, personne ne s'opposera à ce qu'on réduise le nombre des locutions latines. Cependant on aurait tort de ne pas tenir compte de la valeur fonctionnelle de bon nombre d'entre elles. Souvent le latin en dit autant que le français sous une forme plus concise. Avonsnous intérêt à remplacer par des équivalents français - qu'il faudrait parfois créer - numerus clausus, mutatis mutandis, ex cathedra, ex aequo, sine die, ipso facto...? Par contre, il est bien évident que C.Q.F.D. est plus simple et plus clair que Quod erat demonstrandum.

1. Voir * Caractérologie linguistique 3, l'Actualité terminologique, vol. 10 , no 5 (mai 1977), p. 3. 
Les expressions étrangères sont surtout empruntées à l'anglais et relèvent de domaines étrangers au droit. Pour ce qui est des autres sections de cette circulaire, on relève en effet des changements de nature à moderniser le discours sans nuire à son efficacité. Personne ne regrettera le remplacement de sieur, dame, veuve par $M ., M^{\text {me }}, M^{\text {me }}$ veuve $X$... Condamné aux dépens n'a pas besoin d'être renforcé par tous les précédé de en. Donner à bail et prendre à bail, au lieu de louer, suppriment une ambiguité. Partout où l'on a élagué sans nuire au sens, il y a amélioration.

Mais il n'est pas sûr que ledit n'ait pas encore un rôle à jouer dans l'usage actuel, et il appert, simple et bref, a le mérite, qu'ont souvent l'archaïsme et l'emprunt, de se détacher clairement sur l'ensemble de l'énoncé. Il en est de même de nonobstant, qui a plus de relief que malgré.

Somme toute, la Commission a agi sagement en ne recommandant, dans l'ensemble, que des changements qui allègent sans amoindrir.

Nous avons parlé tout au début de l'interaction de ces deux entités linguistiques que sont la nomenclature et le discours d'une spécialité donnée. Il reste à montrer comment elle fonctionne. La nomenclature, pour être opérante, ne peut se passer du discours, et le discours, dans un domaine technique, n'a pas de raison d'être sans la nomenclature. L'un et l'autre sont complémentaires, mais ils sont aussi dans un rapport de supplétion. Il arrive, en effet, que le discours, qui est le plus souple, le plus extensible de ces deux modes d'expression, supplée aux lacunes de la nomenclature. Bloomfield a fait observer que ce qui se dit dans une langue peut toujours se dire dans une autre. Sans doute, mais à condition de faire appel aux ressources du discours. On le voit lorsque le français est en présence de l'anglais ou de l'allemand. Beaucoup des composés de ces deux langues ne peuvent être rendus en français que par des groupes syntaxiques. C'est le cas par exemple de self-defeating ou de counter-productive traduits par qui va à l'encontre du but cherché. De la même façon, quorum relève de la nomenclature et délibérer valablement du discours.

Voici, pour terminer, un exemple moins banal et plus éclairant d'interaction. Pour traduire Edmonton is the northernmost and the highest city in $\mathrm{Ca}$ nada, on dira tout naturellement \& Edmonton est la ville la plus au nord et la plus élevée du Canada ». À ce moment on achoppe à une particularité du système anglais qui a créé entre town et city une dichotomie parfois dimensionnelle, toujours statutaire, et qui n'a pas d'équivalent en français. $\bar{A}$ la réflexion on se rend compte que la souplesse du discours peut permettre de combler cette lacune. Car on peut dire : \& De toutes les grandes villes du Canada, Edmonton est la plus au nord et la plus élevée. \$ Le composé grande ville est un élément du lexique, mais pour l'actualiser de telle façon qu'il fonctionne pleinement au sein de l'énoncé, il a fallu créer un membre de phrase. Cette création est un fait de discours. 
On peut s'étonner que; dans tout ce qui précède, le discours s'oppose à la nomenclature plutôt qu'à la terminologie, et cela mérite un mot d'explication.

La nomenclature n'est que le relevé des termes techniques d'un sujet, termes que le terminologue explique et organise en micro-systèmes. Le discours englobe la nomenclature, la véhicule en quelque sorte, mais il s'en distingue parce qu'il est de nature différente. Comme on l'a fait observer, les termes techniques sont imposés par la matière, alors que, dans l'élaboration du discours, le rédacteur ou traducteur dispose d'une certaine marge, à condition de ne pas trahir le sens.

$\mathrm{Si}$ la dichotomie nomenclature/discours telle qu'elle a été présentée cidessus, est valable, il semble đésirable que la formation du traducteur en tienne compte. Il arrive en effet que celui-ci doive rétablir dans son texte la clarté parfois déficiente de l'original. Pour cela il importe qu'ayant assimilé la terminologie du sujet il soit en mesure d'utiliser les ressources du discours. Notre propos, au cours des pages qui précèdent, a été de montrer la complémentarité de ces deux sortes de ressources qui concourent à l'élaboration du texte.

Le monde de la traduction a découvert récemment l'importance de la recherche en terminologie. Sans se détacher de cette révélation, il aperçoit maintenant la place qu'occupe la rédaction dans le processus de la traduction. Il ne saurait être question de choisir entre ces deux entités, de préférer l'une à l'autre, de les traiter en somme comme des modes successives et passagères, alors que ce qui importe avant tout, c'est de les conjuguer pour la plus grande efficacité de la traduction.

JEAN DARBELNET 